\section{Wasserstoffbasierte Technologien im Verkehr - auch eine Frage von Vertrauen und Vertrautsein}

\author{
von Weert Canzler und Inga Deibel, Berlin*
}

Die zukünftige Energieversorgung beruht auf einem massiven Ausbau und Einsatz von erneuerbaren Energien. Aufgrund ihrer zeitlich und räumlich fluktuierenden Produktion wird die Speicherung ein Schlüsselfaktor für die erfolgreiche Integration der erneuerbaren Energien in die Energiewirtschaft sein. Wasserstoff als Energiespeichermedium bietet sich hierbei als ein Lösungsweg zur kontinuierlichen Bereitstellung erneuerbarer Energien in unterschiedlichen mobilen und stationären Anwendungsfeldern an. Die bislang vorliegenden sozialwissenschaftlichen Studien lassen zu der Akzeptanz der Wasserstoff- und Brennstoffzellentechnologie in der Alltagsanwendung und zu den Erwartungen der Endnutzerinnen und -nutzer im Verkehr nur vage Aussagen zu. Hier soll das 2009 begonnene Projekt HYTRUST neue Erkenntnisse bringen.

\section{Hintergrund und Problemstellung}

Um wasserstoffbasierte Techniken im Mobilitätssektor in Deutschland Wirklichkeit werden zu lassen, wurde 2002 die „Clean Energy Partnership“ (CEP) gegründet. Ziel der Initiative ist es, die Systemfähigkeit von Wasserstoff als zukünftigen Kraftstoff für Automobile zu erproben. Neben der deutschen Bundesregierung sind an der CEP wichtige Unternehmen aus der Automobil- und Energiewirtschaft beteiligt. ${ }^{1}$ In einer Reihe von Erprobungsprojekten konnte mittlerweile gezeigt werden, dass die Betankung von Wasserstofffahrzeugen schnell und sicher möglich ist und dass leistungsfähige Wasserstofffahrzeuge zuverlässig betrieben werden können (vgl. CEP 2010). 2006 wurde von der Bundesregierung darüber hinaus das „Nationale Innovationsprogramm Wasserstoff- und Brennstoffzellentechnologie" (NIP) beschlossen. Im NIP wird u. a. das Ziel der beschleunigten Marktentwicklung von Wasserstoffund Brennstoffzellentechnologien im mobilen, stationären und portablen Bereich genannt. Das bedeutet nicht zuletzt eine Optimierung der Wasserstofftechnologie unter Alltagsbedingungen.
Bei der Einführung mobiler Wasserstofftechnologien handelt es sich um einen technologischen Systemwechsel, der nicht nur mit der Entwicklung einer neuen Antriebstechnologie und dem Aufbau der erforderlichen Infrastruktur zur Produktion und Bereitstellung des Wasserstoffs verbunden ist. Ebenso wichtig ist die Akzeptanz der neuen Technologie. Obwohl die Technologie nach bisherigen Untersuchungen insgesamt positiv wahrgenommen wird, muss aufgrund des geringen Kenntnisstandes in der Bevölkerung und mangelnder direkter Erfahrungswerte nach wie vor mit Vorbehalten gegenüber dem Energieträger Wasserstoff gerechnet werden. Daher ist es notwendig, bereits frühzeitig die, die Akzeptanz beeinflussenden Faktoren, in den verschiedenen gesellschaftlichen Gruppen zu identifizieren. Unter gesellschaftlichen Gruppen ist hierbei nicht nur die allgemeine Öffentlichkeit zu verstehen, sondern auch die in den Unternehmen oder anderen Institutionen beschäftigten Entwickler, Prüfer und Entscheider sowie die meinungsbildenden Akteure in Medien, Interessensverbänden und Politik. Eine solche, breite Akzeptanzuntersuchung mobiler Wasserstofftechnologien hat sich das Projekt HYTRUST, getragen von einem sozialwissenschaftlich geprägten Konsortium $^{2}$ von fünf Forschungsinstituten unter der Federführung des Unabhängigen Instituts für Umweltfragen (UfU), zur Aufgabe gemacht. Das Innovationszentrum für Mobilität und gesellschaftlichen Wandel (InnoZ) trägt mit Fallstudien zur öffentlichen Perzeption und zur Imagebildung des Energieträgers Wasserstoff (sowie der wasserstoffgespeisten Brennstoffzelle) bei.

\section{Nutzungsbilder und Akzeptanz}

Im Mittelpunkt von vier Fallstudien steht die Nutzungsdimension von mobilen Wasserstofftechnologien. Die Nutzerperspektive umfasst die Bedürfnisse, Gewohnheiten und Anforderungen der Menschen sowie alltägliche Abläufe bei der Nutzung von Technik. ${ }^{3}$ Dabei wird davon ausgegangen, dass für eine erfolgreiche Einführung der wasserstoffbasierten Mobilität zum einen eine generelle Akzeptanz in der Bevölkerung und zum anderen das Vertrauen in die technologiebetreibenden Akteure vorhanden sein muss. Akzeptanz und Vertrauen sind nicht selbstverständlich und 
sie sind auch nicht mit der technischen Funktionalität einzelner Elemente wie der Antriebstechnologie, der Speicherung und der Betankungsstationen automatisch gesichert. Vertrauen in bzw. Vertrautheit mit einer neuen Technologie kann nicht als gegeben betrachtet werden. Sie schlägt sich in aller Regel erst nach einer gewissen Zeit in einem habitualisierten Nutzungsverhalten nieder.

Da der Großteil der Bevölkerung nicht direkt in die Entwicklung neuer Technologien einbezogen wird, das Produkt also nicht selbst direkt erfahren kann, und die Komplexität neuer Technologien die eigene Fachkompetenz in der Regel bei Weitem übersteigt, rückt das Vertrauen in die Akteure, die die Wasserstofftechnologie voranbringen, in seiner Relevanz bei der Beurteilung von Technik in den Vordergrund: Die nicht direkt beteiligte Mehrheit der Gesellschaft ist auf die Einschätzung dieser Akteure angewiesen, um Orientierung zu finden. Ein Weg, um den Grad und die Verbreitung von Vertrauen zu identifizieren, besteht in einem prozessbegleitenden Monitoring von Projekten, die die Alltagstauglichkeit von Wasserstoffmobilen und Wasserstoffinfrastruktur demonstrieren sollen.

Deshalb werden im Rahmen von HYTRUST die Fallstudien auf Demonstrationsprojekte fokussiert (Fahrzeugflotten plus Infrastruktur). Empirische Daten werden erhoben, wie Pilotnutzer von wasserstoffbetriebenen Fahrzeugen im Mobilitätssektor (Projektbeteiligte und Kunden) die neue Technologie erleben und welche Chancen, aber auch welche Grenzen sie für die alltägliche Nutzung sehen. Befragt werden neben Einsatzplanern und Projektmanagern insbesondere die Endnutzerinnen und Endnutzer, um deren Motive, Erfahrungen, Emotionen (und insbesondere ihr Vertrauen oder Misstrauen) in die Wasserstofftechnologie zu erfahren. Neben Sicherheit und Risikoabschätzung sind weiterhin spezifische Problem des Handlings und der Routinefähigkeit von Interesse. Diese Nutzereinschätzungen - so lautet die Hypothese - sind immer vor dem Hintergrund bisheriger Erfahrungen mit konventionellen Energieträgern im Verkehr zu beurteilen. Außerdem ist $\mathrm{zu}$ untersuchen, ob und in welchem Ausmaß das spezifische Design der verschiedenen Demonstrationsprojekte einen Unterschied ausmacht.

Methodisch werden teilnehmende Beobachtungen mit Tiefeninterviews mit Teilnehmerinnen und Teilnehmern der Demonstrationsvorhaben verknüpft. In diesen Interviews können insbesondere die Motive und Emotionen sowie die Routinebildung (bzw. ihr Ausbleiben) bei den Befragten sowohl direkt als auch indirekt adressiert werden. Neben Einstellungs- und Bewertungsfragen werden alltagsstrukturierende Handlungs- und Mobilitätsmuster abgefragt. So kann es gelingen, Aussagen über die Integration von Techniknutzungen in habitualisiertes Handeln zu treffen.

\section{Erste Ergebnisse und Aussichten}

Nach Abschluss der ersten beiden Fallstudien zeichnet sich Folgendes ab: Insgesamt findet eine mögliche Einführung der Wasserstofftechnologie im Mobilitätssektor wohlwollende Unterstützung. Wasserstoff hat bei fast allen befragten Personen ein positives und umweltfreundliches Image, da es als saubere Alternative zu fossilen Kraftstoffen wahrgenommen wird. Es besteht bei den Befragten eine hohe Aufgeschlossenheit gegenüber regenerativen Energien, und Wasserstoff kann von einem Imagetransfer profitieren. Viele Interviewpartner halten Wasserstoff im Verkehrssektor für zukunftsfähig, sofern die mit ihr verbundenen Technologien bezahlbar sind. Die Befragten scheinen darüber hinaus der Technik und den sie entwickelnden Akteuren überwiegend zu vertrauen. So sehen beispielsweise die befragten Personen keine besonderen Gefahren, wenn in Wohnortnähe eine Wasserstofftankstelle anstelle einer herkömmlichen Tankstelle installiert würde. Auf einzelne Akteursgruppen bezogen zeigt sich, dass die Wissenschaft als Akteur bei den Befragten einen Vertrauensbonus in Sachen Kompetenz und Umweltorientierung genießt. Auch die potenziell beteiligten Unternehmen können mit Wohlwollen rechnen: Wenn im Zuge der Bemühungen zur Einführung dieser Technologie mehr und mehr große Unternehmen als erkennbare Akteure auftreten, kann das unter Umständen ihr Image verbessern. Zugetraut wird ihnen zumindest die Fähigkeit zur Umsetzung dieses umfassenden Schrittes in Richtung einer nachhaltigen Entwicklung des motorisierten Individualverkehrs und der effizienten Nutzung erneuerbarer Energien.

Mit Blick auf die Akteure der Wasserstofftechnologie-Initiative lässt sich bereits jetzt die 
Empfehlung formulieren, dass v. a. folgende Punkte verstärkt Berücksichtigung finden sollten: Das Vertrauen potenzieller Nutzer in die Technologie wird nach Ansicht von befragten Projektprotagonisten mehrheitlich über eine Demonstration der Technologie sowohl in Form von Infrastruktur als auch durch Anwendung (z. B. Sichtbarkeit von Fahrzeugen im öffentlichen Raum) erreicht. Die Nutzungs- und Kaufbereitschaft der Wasserstofftechnologie hängt dabei im Wesentlichen von der zielgerichteten, ehrlichen und aufklärenden Kommunikation ab. Derzeitige Unsicherheiten bestehen hinsichtlich der Anschaffungskosten von Fahrzeugen, ökologischer Nachhaltigkeit des Wasserstoffs und der Einordnung von Wasserstoff in den Kontext der Elektromobilität. Um einer reservierten Haltung gegenüber der Technologie aufgrund fehlender Erfahrungswerte zu begegnen, sollte der Schwerpunkt im Einsatz von Flottenfahrzeugen auf Basis von kurzen Leasingverträgen mit integrierten Servicepaketen liegen.

Wie geht es im HYTRUST-Projekt weiter? Im Fokus der nächsten Fallstudien stehen verstärkt die Erwartungen von Pilotanwendern und von potenziellen Nutzern an die Wasserstoff- und Brennstoffzellentechnologie. Die Hypothese lautet, dass die Erwartungen zum einen davon beeinflusst werden, ob und wie Erfahrungen in der Nutzung von wasserstoffbasierter Mobilität vorliegen und wie sich die Rahmenbedingungen einer Wasserstoffnutzung im Verkehr entwickeln werden. In den bisherigen Interviews wurden immer wieder die Senkung der Beschaffungs- und Unterhaltungskosten von Fahrzeugen sowie die Schaffung einer Infrastruktur genannt. Staatliche Instrumente werden oftmals als Basis für die erfolgreiche Einführung der Technologie gesehen. Diese beziehen sich dabei auf die Phase der Markteinführung der Technologie, z. B. durch die Minimierung von rechtlichen Hemmnissen und der Setzung positiver Anreize zur Förderung regenerativer Energien. Für die erfolgreiche Implementierung der Technologie ist außerdem von grundlegender Bedeutung, ob die Fahrzeughersteller bereit sind, ein breites Angebot zu schaffen. Durch die Gründung und Umsetzung strategischer Allianzen kann der Aufbau der Infrastruktur und die Einführung von Fahrzeugen in einem breiter wahrnehmbaren Umfang vereinfacht werden. Erste Ergebnisse aus dem
Forschungsprojekt zeigen, dass durchaus günstige Grundvoraussetzungen bestehen, um Wasserstoff im Mobilitätssektor einzuführen.

\section{Anmerkungen}

* Weert Canzler arbeitet am Wissenschaftszentrum Berlin für Sozialforschung (WZB) und Inga Deibel im Innovationszentrum für Mobilität und gesellschaftlichen Wandel.

1) Dazu gehören: BMW Group, Berliner Verkehrsbetriebe (BVG), Daimler AG, Ford, GM/Opel, Hamburger Hochbahn, Linde, Shell, StatoilHydro, TOTAL, Toyota, Vattenfall Europe und die Volkswagen AG; siehe auch: http://www.cleanenergypartnership.de.

2) Das von der Nationalen Organisation Wasserstoffund Brennstoffzellentechnologie (NOW) geförderte und auf fünf Jahre angelegte Projekt startete im Sommer 2009. Dem Konsortium gehören an: Unabhängiges Institut für Umweltfragen (UfU), PotsdamInstitut für Klimafolgenforschung, ECF - European Climate Forum, Institut für Transportation Design, Innovationszentrum für Mobilität und gesellschaftlichen Wandel (InnoZ) und Spilett New Technologies GmbH; siehe auch: http://www.hytrust.de.

3) Unter Nutzerperspektive wird im Rahmen dieses Projekts nicht nur die Sichtweise der Bevölkerung verstanden, sondern die Wahrnehmungsperspektiven aller am Innovationsprozess beteiligten Akteure. Auf den epochalen Shift auf die Nutzungsseite bei Innovation in spätindustriellen Gesellschaften hat bereits Wengenroth (2001) hingewiesen.

\section{Literatur}

CEP - Clean Energy Partnership, 2010: Wasserstoff bewegt - kommen Sie mit! CEP Phase II \& III, Stand 21.08.2010; http://www.cleanenergypartnership.de/ uploads/tx_cepdownloadsv2/20100921_CEP_Broschuere_low_02.pdf (download 7.1.11)

Wengenroth, U., 2001: Vom Innovationssystem zur Innovationskultur. In: Abele, J.; Barkleit, G.; Hänseroth, T. (Hg): Innovationskulturen und Fortschrittserwartungen im geteilten Deutschland. Köln, S. 23-32

\section{Kontakt}

Dr. Weert Canzler

Wissenschaftszentrum Berlin für Sozialforschung (WZB)

Forschungsgruppe Wissenschaftspolitik

Reichpietschufer 50, 10785 Berlin

Tel: + 49 (0) 30 / 25491 - 202

E-Mail: canzler@wzb.eu

《॥ 